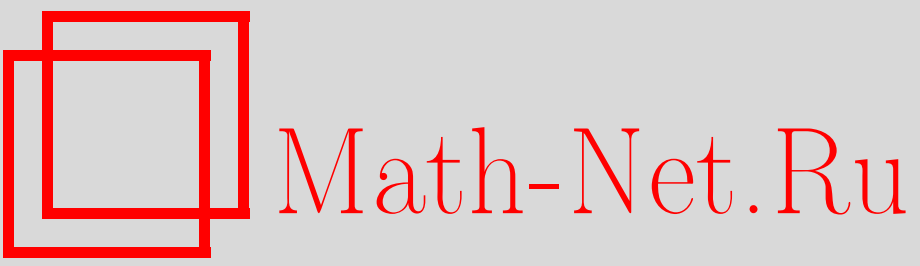

В. В. Абрамов, Е. Ю. Лискина, К вопросу о периодическом решении обыкновенного дифференциального уравнения второго порядка, Итоги науки и техн. Сер. Соврем. мат. и ее прил. Темат. обз., 2020, том 185, 13-18

DOI: https://doi.org/10.36535/0233-6723-2020-185-13-18

Использование Общероссийского математического портала Math-Net.Ru подразумевает, что вы прочитали и согласны с пользовательским соглашением

http://www.mathnet.ru/rus/agreement

Параметры загрузки:

IP: 18.234 .156 .22

26 апреля 2023 г., 08:05:38 


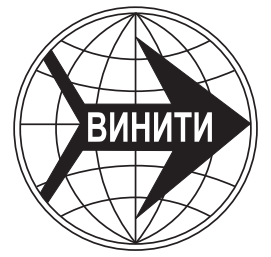

ИТОГИ НАУКИ И ТЕХНИКИ.

Современная математика и ее приложения.

Тематические обзоры.

Том 185 (2020). C. $13-18$

DOI: $10.36535 / 0233-6723-2020-185-13-18$

УДК 517.925 .42

\title{
К ВОПРОСУ О ПЕРИОДИЧЕСКОМ РЕШЕНИИ ОБЫКНОВЕННОГО ДИФФЕРЕНЦИАЛЬНОГО УРАВНЕНИЯ ВТОРОГО ПОРЯДКА
}

\author{
(C) 2020 г. \\ В. В. АБРАМОВ, Е. Ю. ЛИСКИНА
}

\begin{abstract}
АннотАция. Рассматривается дифференциальное уравнение, содержащее формы первого и второго порядка относительно фазовой переменной и ее производной с постоянными коэффициентами, а также периодическую неоднородность. Решается задача о существовании асимптотически устойчивого по Ляпунову периодического решения. Для проведения рассуждений применен метод построения положительно инвариантной прямоугольной области. Признаки существования периодического решения сформулированы в терминах свойств изоклин. Рассмотрены случаи, когда изоклина нуля является невырожденной кривой второго порядка.
\end{abstract}

Ключевъе слова: дифференциальное уравнение второго порядка, качественная теория, периодическое решение, устойчивость, нелинейный осциллятор.

\section{ON PERIODIC SOLUTIONS OF A SECOND-ORDER ORDINARY DIFFERENTIAL EQUATION}

\author{
(c) 2020 V. V. ABRAMOV, E. YU. LISKINA
}

\begin{abstract}
We consider a differential equation containing first- and second-order forms with respect to the phase variable and its derivative with constant coefficients and a periodic inhomogeneity. Using the method of constructing a positively invariant rectangular domain, we examine the existence of a asymptotically stable (in the Lyapunov sense) periodic solution. Criteria for the existence of a periodic solution are formulated in terms of properties of isoclines. We consider cases where the zero isocline is a nondegenerate second-order curve.
\end{abstract}

Keywords and phrases: second-order differential equation, qualitative theory, periodic solution, stability, nonlinear oscillator.

AMS Subject Classification: 34C05, 34C25, 34D20

1. Введение. Рассмотрим дифференциальное уравнение

$$
\ddot{x}+a x+b \dot{x}+c(t)+a x^{2}+\beta x \dot{x}+\gamma(\dot{x})^{2}=0,
$$

в котором коэффициенты $a, b, \alpha, \beta, \gamma$ постоянны, $c(t)$ - ограниченная $T$-периодическая функция. Установим признаки существования устойчивого $T$-периодического решения уравнения (1).

Уравнение вида (1) часто используется в качестве модели нелинейного осциллятора и относится к классу обобщенных уравнений типа Рэлея. Классические результаты исследований по проблеме существования периодических решений уравнений типа Льенара, Рэлея и их обобщений изложены в [1]. В данной работе применим метод канонических областей, в основе которого лежит следующее утверждение (см. [2]). 
Теорема 1. Пусть система $\dot{x}=f(t, x)$ имеет $T$-периодическую по $t$ правую часть, обладает свойством существования и единственности решения задачи Коши во множестве $\mathbb{R} \times D$. Если существует выпуклый компакт $\Omega \subset D$, ограниченный гладкими кривыми $\Phi_{i}(x)=0$, на которых имеют место оценки $\left(\operatorname{grad} \Phi_{i}(x), f(t, x)\right) \leqslant 0, i=\overline{1, m}$, то система $\dot{x}=f(t, x)$ имеет $T$-периодическое решение с начальным значением в Int $\Omega$.

Любую область $\Omega$, удовлетворяющую теореме 1 , будем называть канонической.

2. Вспомогательные результаты. Сведем уравнение (1) к эквивалентной нормальной системе второго порядка. Пусть $\dot{x}=k x-y, k$-параметр. Тогда $\ddot{x}=k \dot{x}-\dot{y}$. Подставив $\dot{x}, \ddot{x}$ в уравнение (1), получим соотношение

$$
k^{2} x-k y-\dot{y}+a x+b k x-b y+c(t)+\alpha x^{2}+\beta x y+\gamma k^{2} x^{2}+\gamma y^{2}-2 \gamma k x y=0,
$$

в силу которого

$$
\dot{y}=x^{2}\left(\alpha+\beta k+\gamma k^{2}\right)+y^{2} \gamma+x\left(a+b k+k^{2}\right)-x y(\beta+2 \gamma k)-y(k+b)+c(t) .
$$

Далее будем предполагать, что параметр $k$ удовлетворяет условию $\beta+2 \gamma k=0$. При этом уравнению (1) соответствует система

$$
\left\{\begin{array}{l}
\dot{x}=f_{1}(x, y)=k x-y, \\
\dot{x}=f_{2}(t, x, y)=x^{2}\left(\alpha-\gamma k^{2}\right)+\gamma y^{2}+x\left(a+b k+k^{2}\right)-y(k+b)+c(t) .
\end{array}\right.
$$

Итак, задача о периодическом решении уравнения (1) сводится к задаче о периодическом решении системы (2).

Установим необходимые условия существования периодического решения. Для этого рассмотрим расположение изоклины бесконечности $I^{\infty}: \dot{x}=0$ и подвижной изоклины нуля $I_{t}^{\infty}: \dot{x}=0$ системы (2).

При каждом $t$ изоклина $I^{\infty}\left(I_{t}^{0}\right)$ делит плоскость $x O y$ на две части: внутреннюю, в которой $\dot{x}<0(\dot{y}<0)$, и внешнюю, в которой $\dot{x}>0(\dot{y}>0)$. Эти части обозначим соответственно $X^{\text {int }}$, $X^{\mathrm{ext}},\left(Y_{t}^{X^{\mathrm{int}}}, Y_{t}^{\mathrm{ext}}\right)$. Построим для изоклины $I_{t}^{0}$ соответствующие неподвижные (стационарные) внутреннюю и внешнюю части фазовой плоскости:

$$
Y^{\mathrm{ext}}=\left\{(x, y): f_{2}(t, x, y)>0, t \in \mathbb{R}\right\}, \quad Y^{\mathrm{int}}=\left\{(x, y): f_{2}(t, x, y)<0, t \in \mathbb{R}\right\} .
$$

Очевидно, при всех $t \in \mathbb{R}$ изоклины удовлетворяют условиям

$$
I^{\infty}=X=\partial X=\partial X^{\text {int }} \cup \partial X^{\text {ext }}, \quad I_{t}^{0} \subset Y, \quad \partial Y=\partial Y^{\text {int }} \cup \partial Y^{\text {ext }},
$$

где $Y$ - область, содержащая все изоклины нуля $I_{t}^{0}$. Так как в каждой из областей $X^{\mathrm{int}}, X^{\text {ext }}$, $Y^{\text {int }}, Y^{\text {ext }}$ компоненты векторного решения системы (2) строго монотонны, то траектория периодического решения системы (2) не может целиком лежать ни в одной из этих областей. Поэтому справедливо следующее утверждение о локализации канонической области.

Лемма 1. Если область $\Omega$ является канонической для системы (2), то имеет место включение $\Omega \supset X \cap Y$.

Запишем систему (2) в векторной форме $\dot{z}=f(z)+\operatorname{colon}(0, c(t))$,

$$
z=\left(\begin{array}{l}
x \\
y
\end{array}\right), \quad f(z)=\left(\begin{array}{c}
k x-y \\
x^{2}\left(\alpha-\gamma k^{2}\right)+\gamma y^{2}+x\left(a+b k+k^{2}\right)-y(k+b)
\end{array}\right) .
$$

Выберем какие-либо решения $z_{1}=z_{1}(t), z_{2}=z_{2}(t)$ системы $(2)$. Для функции $V\left(z_{1}, z_{2}\right)=\left(z_{1}-z_{2}\right)^{2}$ вычислим производную в силу системы (2). По формуле Лагранжа получим

$$
\dot{V}\left(z_{1}, z_{2}\right)=2\left(z_{1}-z_{2}\right)^{T} F(\tilde{z})\left(z_{1}-z_{2}\right)
$$

где

$$
F(\tilde{z})=\frac{1}{2}\left(\frac{\partial f(\tilde{z})}{\partial z}+\left(\frac{\partial f(\tilde{z})}{\partial z}\right)^{T}\right)
$$


- эрмитова компонента матрицы Якоби, $\tilde{z}$ - некоторый вектор, выбор которого зависит от $z_{1}$ и $z_{2}$.

Предположим, что область $\Lambda$ является положительно инвариантной для системы $(2)$. Произвольно выберем $z_{1}(0)$ и $z_{2}(0)$. Тогда $z_{1}(t), z_{2}(t) \in \Lambda$ для любого $t \geqslant 0$. Допустим, что $z_{1}$ и $z_{2}-$ различные $T$-периодические решения с траекториями, лежащими в положительно инвариантной канонической области $\Omega$. Если $\dot{V}\left(z_{1}, z_{2}\right)<0$ при $t \geqslant 0$, то $V\left(z_{1}, z_{2}\right)$ строго убывает при $t \geqslant 0$. При этом

$$
z_{1}(0) \neq z_{2}(0), \quad V\left(z_{1}(0), z_{2}(0)\right)>V\left(z_{1}(T), z_{2}(T)\right)
$$

т.e.

$$
\left\|z_{1}(0)-z_{2}(0)\right\|_{2}>\left\|z_{1}(T)-z_{2}(T)\right\|_{2}
$$

Значит, функция $z(t)=z_{1}(t)-z_{2}(t)$ не является $T$-периодической. Таким образом, если $\dot{V}\left(z_{1}, z_{2}\right)<$ 0 при $t \geqslant 0$ в области $\Omega$, то в этой области содержится единственная траектория периодического решения системы $(2)$.

Более того, если $\dot{V}\left(z_{1}, z_{2}\right)<0$ при $t \geqslant 0$, где $z_{1}(t) \in \Omega-T$-периодическое решение системы $(2)$, $z_{1}(t), z_{2}(t) \in \Omega$ - произвольно выбранное решение системы $(2)$, то имеет место оценка

$$
V\left(z_{1}(0), z_{2}(0)\right)>V\left(z_{1}(T), z_{2}(T)\right)
$$

Значит, оператор сдвига по траекториям отображает окрестность начального значения $z_{1}(0)$ в себя. Отсюда по индукции следует устойчивость периодического решения $z_{1}$ (см. [2]).

Итак, если $F(z)<0$ в положительно инвариантной области $\Omega$, то $\dot{V}\left(z_{1}, z_{2}\right)<0$ при $t \geqslant 0$ для любой пары решений с начальными значениями $\Omega$. При этом в области $\Omega$ лежит единственная траектория устойчивого периодического решения системы $(2)$.

Для системы (2) имеем

$$
F(z)=\left[\begin{array}{cc}
k & \frac{2 x\left(\alpha-\gamma k^{2}\right)+\left(a+b k+k^{2}\right)-1}{2} \\
\frac{2 x\left(\alpha-\gamma k^{2}\right)+\left(a+b k+k^{2}\right)-1}{2} & 2 \gamma y-(k+b)
\end{array}\right] .
$$

Согласно критерию Сильвестра условие $F(z)<0$ в $\Omega$ равносильно системе оценок для главных миноров:

$$
F(z)=\left\{\begin{array}{l}
\Delta_{1}=k<0 \\
\Delta_{2}(x, y)=(2 \gamma y-(k+b))-\frac{\left(2 x\left(\alpha-\gamma k^{2}\right)+\left(a+b k+k^{2}\right)-1\right)^{2}}{4}>0 .
\end{array}\right.
$$

Поэтому всюду далее, решая поставленную задачу, будем полагать, что выполняется условие

$$
k<0 \text {. }
$$

При этом из принятого ранее условия $\beta+2 \gamma k=0$ следует, что знаки коэффициентов $\beta, \gamma$ одинаковы.

В данной работе границу канонической области $\partial \Omega$ будем искать в виде прямоугольника $A B C D$ :

$$
\begin{array}{ll}
A B: \Phi_{1}(x, y)=y_{1}-y=0 ; & \operatorname{grad} \Phi_{1}=(0 ;-1) ; \quad B C: \Phi_{2}(x, y)=x-x_{2}=0 ; \quad \operatorname{grad} \Phi_{2}=(1 ; 0) \\
A D: \Phi_{3}(x, y)=x_{1}-x=0 ; \quad \operatorname{grad} \Phi_{3}=(-1 ; 0) ; \quad B C: \Phi_{4}(x, y)=y-y_{2}=0 ; \quad \operatorname{grad} \Phi_{4}=(0 ; 1)
\end{array}
$$

При этом для применения теоремы 1 требуется проверка неравенств с нелинейными членами того же порядка, что и в правой части рассматриваемой системы. Кроме того, для проверки условия устойчивости достаточно рассмотреть вершины прямоугольника. В таком случае теорему 1 можно сформулировать в следующем виде. 
Теорема 2. Пусть правая часть системь (2) на границе $A B C D$ удовлетворяет условиям

$$
\left\{\begin{array}{l}
f_{2}(t, x, y) \geqslant 0, \quad \text { если } \Phi_{1}=0, \\
f_{1}(t, x, y) \geqslant 0, \quad \text { если } \Phi_{2}=0, \\
f_{1}(t, x, y) \geqslant 0, \quad \text { если } \Phi_{3}=0 \\
f_{2}(t, x, y) \geqslant 0, \quad \text { если } \Phi_{4}=0 .
\end{array}\right.
$$

Кроме того, в вершинах прямоугольника $A B C D$ справедлива оченка $\Delta_{2}(x, y)>0$. Тогда система (2) имеет устойчивое T-периодическое решение с начальным значением внутри $A B C D$.

3. Основные результаты. Рассмотрим невырожденные частные случаи соответствия изоклины $I_{t}^{0}$ одной из стандартных форм для кривых второго порядка, когда система (2) не является линейной.

Будем использовать обозначения

$$
c_{1}=\inf _{0, T} c(t), \quad c_{2}=\sup _{0, T} c(t) .
$$

Допустим, что $\gamma \neq 0, \alpha-\gamma k^{2} \neq 0$. Выделим полные квадраты в уравнении для $I_{t}^{0}$ :

$$
\left(\alpha-\gamma k^{2}\right)\left(x-x_{0}\right)^{2}+\gamma\left(y-y_{0}\right)^{2}=m-c(t),
$$

где

- центр кривой,

$$
\left(x_{0}, y_{0}\right)=\left(\frac{-a-b k-k^{2}}{2\left(\alpha-\gamma k^{2}\right)} ; \frac{k+b}{2 \gamma}\right)
$$

$$
m=\frac{\left(a+b k+k^{2}\right)^{2}}{2\left(\alpha-\gamma k^{2}\right)}+\frac{(k+b)^{2}}{4 \gamma}
$$

Предположим, что справедливы оценки

$$
\gamma<0, \quad \alpha-\gamma k^{2}>0, \quad c_{1}>m
$$

Тогда кривая (4) - это гипербола с полуосями

$$
\sqrt{\frac{c(t)-m}{\alpha-\gamma k^{2}}}, \quad \sqrt{\frac{m-c(t)}{\gamma}} .
$$

Для применения теоремы 2 потребуем, чтобы пересекались линии $I^{\infty}: y=k x$ и

где

$$
I_{1}^{0}: \frac{\left(y-y_{0}\right)^{2}}{s_{1}^{2}}-\frac{\left(x-x_{0}\right)^{2}}{q_{1}^{2}}=1, \quad y>y_{0},
$$

Таким образом, дискриминант уравнения

$$
q_{1}=\sqrt{\frac{\left|m-c_{2}\right|}{\alpha-\gamma k^{2}}}, \quad s_{1}=\sqrt{\frac{\left|m-c_{2}\right|}{|\gamma|}} .
$$

$$
\frac{\left(k x-y_{0}\right)^{2}}{s_{1}^{2}}-\frac{\left(x-x_{0}\right)^{2}}{q_{1}^{2}}=1
$$

должен быть положительным. Это условие выполнено, если справедливо неравенство

$$
\left(k x_{0}-y_{0}\right)^{2}+\left(k^{2} q_{1}^{2}-s_{1}^{2}\right)>0 .
$$

Итак, при условии (7) линии $I^{\infty}$ и $I_{1}^{0}, y>y_{0}$, пересекаются в некоторых точках $\left(h_{1}, k h_{1}\right),\left(h_{2}, k h_{2}\right)$, где $h_{1}, h_{2}$ - корни уравнения (6), $h_{1}<h_{2}$.

Для гиперболы $I_{2}^{0}$ рассмотрим вершину $\left(x_{0}, y_{0}+s_{2}\right), s_{2}=\sqrt{\left|m-c_{1}\right| /|\gamma|}$. При условии (7) линии $I^{\infty}$ и $I_{2}^{0}$ пересекаются дважды. Рассмотрим их точку пересечения с большей абсциссой $l$. Выберем прямые

$$
A B: y=y_{l}=\left[\begin{array}{ll}
k l, & l \leqslant x_{0}, \\
y_{0}+s_{2}, & l>x_{0},
\end{array} \quad B C: x=x_{2}=\frac{y_{1}}{k} .\right.
$$


При этом $A B \subset Y^{\mathrm{ext}}, B C \subset X^{\mathrm{int}}$.

Вычислим ординату $y_{2}$ точки пересечения прямой $B C$ с линией $I_{1}^{0}$. Если выполняется условие

$$
k h_{2} \leqslant y_{2} \leqslant k h_{1},
$$

то выберем прямые

$$
C D: y=y_{2}, \quad D A: x=x_{1}=\frac{y_{2}}{k} .
$$

В прямоугольнике $\Omega$, ограниченном линиями (8), (10), выполняется условие устойчивости, если имеют место неравенства

$$
\Delta_{2}\left(x_{1}, y_{1}\right)>0, \quad \Delta_{2}\left(x_{2}, y_{1}\right)>0 .
$$

Итак, согласно теореме 2 справедливо следующее утверждение.

Теорема 3. Если выполняются условия (3), (5), (7), (9), (11), то система (2) имеет устойчивое Т-периодическое решение, траектория которого лежит внутри области, ограниченной прямыми (8), (10).

Аналогично рассматриваются другие случаи, когда изоклина нуля является гиперболой, а также случаи, когда линия (4) - это эллипс или парабола.

Предположим, что справедливы оценки

$$
\gamma>0, \quad \alpha-\gamma k^{2}>0, \quad c_{2}<m .
$$

Тогда при любом $t \in \mathbb{R}$ изоклина $I_{t}^{0}$, заданная уравнением (4), - это эллипс с полуосями

$$
\sqrt{\frac{m-c(t)}{\alpha-\gamma k^{2}}}, \quad \sqrt{\frac{m-c(t)}{\gamma}}
$$

При этом величины

$$
q_{1}=\inf _{[0, T]}\left(\sqrt{\frac{m-c(t)}{\alpha-\gamma k^{2}}}\right)=\sqrt{\frac{m-c_{2}}{\alpha-\gamma k^{2}}}, \quad s_{1}=\inf _{[0, T]}\left(\sqrt{\frac{m-c(t)}{\gamma}}\right)=\sqrt{\frac{m-c_{2}}{\gamma}}
$$

- полуоси границы $I_{t}^{0}$ области $Y^{\text {int }}$, которая содержит центр $\left(x_{0}, y_{0}\right)$ эллипса, а величины

$$
q_{2}=\sup _{[0, T]}\left(\sqrt{\frac{m-c(t)}{\alpha-\gamma k^{2}}}\right)=\sqrt{\frac{m-c_{1}}{\alpha-\gamma k^{2}}}, \quad s_{2}=\sup _{[0, T]}\left(\sqrt{\frac{m-c(t)}{\gamma}}\right)=\sqrt{\frac{m-c_{1}}{\gamma}}
$$

- полуоси границы $I_{2}^{0}$ области $Y^{\mathrm{ext}}$. Для применения теоремы 2 и в этом случае потребуем, чтобы линии $I^{\infty}$ и $I_{t}^{0}$ пересекались дважды в некоторых точках $\left(h_{1}, k h_{1}\right),\left(h_{2}, k h_{2}\right), h_{1}<h_{2}$. Это возможно при условии

$$
\left(k^{2} q_{1}^{2}+s_{1}^{2}\right)-\left(k x_{0}-y_{0}\right)^{2}>0 .
$$

При условии (13) линии $I^{\infty}$ и $I_{2}^{0}$ также пересекаются дважды. Допустим, что $l$ - наибольшая из абсцисс их точек пересечения. Выберем прямые

$$
A B: y=y_{l}=\left[\begin{array}{ll}
k l, & l \leqslant x_{0}, \\
y_{0}+s_{2}, & l>x_{0},
\end{array} \quad B C: x=x_{2}=\frac{y_{1}}{k} .\right.
$$

Пусть $y_{2}$ - ордината точки пересечения прямой $B C$ с изоклиной $I_{1}^{0}$. Если выполняется условие

$$
k h_{2} \leqslant y_{2} \leqslant k h_{1},
$$

то выберем прямые

$$
C D: y=y_{2}, \quad D A: x=x_{1}=\frac{y_{2}}{k} .
$$

В прямоугольнике $\Omega$, ограниченном линиями (14), (18), выполняется условие устойчивости, если справедливы неравенства

$$
\Delta_{2}\left(x_{i}, y_{j}\right)>0 ; \quad i, j=1,2 .
$$

Итак, по теореме 2 справедливо следующее утверждение. 
Теорема 4. Если выполняются условия (3), (12), (13), (15), (17), то система (2) имеет устойчивое Т-периодическое решение, траектория которого лежит внутри области, ограниченной прямыми (14), (17).

Предположим, что в системе (3)

$$
\gamma=0, \quad \alpha>0, \quad k+b>0 .
$$

Тогда при любом значении $t$ изоклина нуля - это парабола

$$
y=\frac{\alpha}{k+b} x^{2}+\frac{a+b k+k^{2}}{k+b} x+\frac{c(t)}{k+b},
$$

ветви которой направлены вверх, вершина имеет абсциссу $x_{0}=\left(-a-b k-k^{2}\right) /(2 \alpha)$. При условии (18) область $Y^{\mathrm{ext}}$ расположена ниже параболы (19), а область $Y^{\mathrm{int}}$-выше. Неподвижные изоклины нуля $I_{1}^{0}, I_{2}^{0}$ имеют ординаты вершин соответственно

$$
s_{1}=\frac{c_{2}}{k+b}, \quad s_{2}=\frac{c_{1}}{k+b} .
$$

Допустим, что справедливо неравенство

$$
a^{2}-4 \alpha c_{2}>0 .
$$

Тогда линии $I^{\infty}$ и $I_{1}^{0}$ имеют две точки пересечения $\left(h_{1}, k h_{1}\right),\left(h_{2}, k h_{2}\right), h_{1}<h_{2}$.

Выберем прямые

$$
A B: y=y_{l}=\left[\begin{array}{ll}
k l, & l \leqslant x_{0}, \\
y_{0}+s_{2}, & l>x_{0},
\end{array} ; \quad B C: x=x_{2}=\frac{y_{1}}{k} .\right.
$$

где $l$ - большая из абсцисс точек пересечения линии $I^{\infty}$ и $I_{2}^{0}$. Вычислим ординату $y_{2}$ точки пересечения $B C$ и $I_{1}^{0}$. При условии

$$
k h_{2} \leqslant y_{2} \leqslant k h_{1}
$$

выберем прямые

$$
C D: y=y_{2}, \quad D A: x=x_{1}=\frac{y_{2}}{k} .
$$

В построенном прямоугольнике $A B C D$ выполняется условие устойчивости, если справедливы оценки

$$
\left\{\begin{array}{l}
\Delta_{2}\left(x_{1}, y_{1}\right)>0 \\
\Delta_{2}\left(x_{2}, y_{1}\right)>0
\end{array}\right.
$$

Итак, справедливо следующее утверждение.

Теорема 5. Если выполняются условия (3), (18), (20), (22), (24), то система (2) имеет устойчивое Т-периодическое решение, траектория которого лежит внутри области, ограниченной прямыми (21), (23).

\section{СПИСОК ЛИТЕРАТУРЫ}

1. Рейссиг P., Сансоне Г., Конти Р. Качественная теория нелинейных дифференциальных уравнений. М.: Мир, 1974.

2. Красносельский M. А. Оператор сдвига по траекториям дифференциальных уравнений. - М.: Наука, 1966.

Абрамов Владимир Викторович

Рязанский государственный университет им. С. А. Есенина

E-mail: v.abramov@365.rsu.edu.ru

Лискина Екатерина Юрьевна

Рязанский государственный университет им. С. А. Есенина

E-mail: e.liskina@365.rsu.edu.ru 